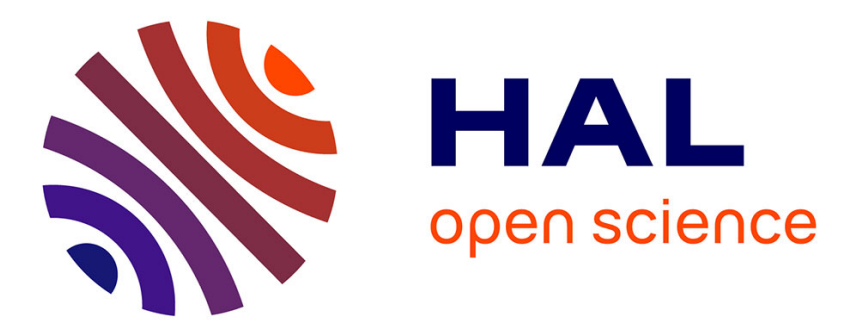

\title{
Layer-resolved imaging of domain wall interactions in magnetic tunnel junction-like trilayers
}

Jan Vogel, Salia Cherifi, Stefania Pizzini, Fabien Romanens, Julio Camarero, Frédéric Petroff, Stefan Heun, Andrea Locatelli

\section{- To cite this version:}

Jan Vogel, Salia Cherifi, Stefania Pizzini, Fabien Romanens, Julio Camarero, et al.. Layer-resolved imaging of domain wall interactions in magnetic tunnel junction-like trilayers. Journal of Physics: Condensed Matter, 2007, 19, pp.476204. 10.1088/0953-8984/19/47/476204 . hal-00133628v2

\section{HAL Id: hal-00133628 \\ https://hal.science/hal-00133628v2}

Submitted on 8 Nov 2007

HAL is a multi-disciplinary open access archive for the deposit and dissemination of scientific research documents, whether they are published or not. The documents may come from teaching and research institutions in France or abroad, or from public or private research centers.
L'archive ouverte pluridisciplinaire HAL, est destinée au dépôt et à la diffusion de documents scientifiques de niveau recherche, publiés ou non, émanant des établissements d'enseignement et de recherche français ou étrangers, des laboratoires publics ou privés. 


\title{
Layer-resolved imaging of domain wall interactions in magnetic tunnel junction-like trilayers
}

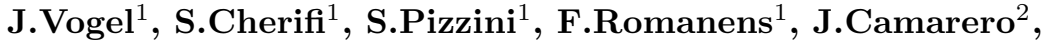 \\ F.Petroff ${ }^{3}$ and S.Heun ${ }^{4}$ and A.Locatelli ${ }^{5}$ \\ ${ }^{1}$ Institut Néel, CNRS \& Université Joseph Fourier, BP166, 38042 Grenoble \\ Cedex 9, France \\ 2 Dpto. Física de la Materia Condensada, Universidad Autónoma de Madrid, \\ 28049 Madrid, Spain \\ ${ }^{3}$ Unité Mixte de Physique CNRS/Thales, Route Départementale 128, 91767 \\ Palaiseau Cedex, France and Université Paris-Sud XI, 91405 Orsay Cedex, \\ France \\ ${ }^{4}$ Laboratorio Nazionale TASC, S.S. 14, km 163.5 in Area Science Park, 34012 \\ Basovizza, Trieste, Italy \\ ${ }^{5}$ Sincrotrone Trieste S.C.p.A., S.S. 14, km 163.5 in Area Science Park, 34012 \\ Basovizza, Trieste, Italy \\ PACS numbers: $75.60 . \mathrm{Ch}, 75.70 . \mathrm{Kw}, 07.85 . \mathrm{Tt}$
}

\begin{abstract}
We have performed a layer-resolved, microscopic study of interactions between domain walls in two magnetic layers separated by a nonmagnetic one, using high-resolution x-ray photoemission electron microscopy. Domain walls in the hard magnetic Co layer of a $\mathrm{Co} / \mathrm{Al}_{2} \mathrm{O}_{3} / \mathrm{FeNi}$ trilayer with in-plane uniaxial anisotropy strongly modify the local magnetization direction in the soft magnetic FeNi layer. The stray fields associated to the domain walls lead to an antiparallel coupling between the local Co and FeNi moments. For domain walls parallel to the easy magnetization axis this interaction is limited to the domain wall region itself. For strongly charged (head-on or tail-to-tail) walls, the antiparallel coupling dominates the interaction over radial distances up to several micrometers from the centre of the domain wall.
\end{abstract}




\section{Introduction}

Magnetic trilayer systems consisting of two thin ferromagnetic (FM) layers separated by a non-magnetic (NM) spacer layer exhibit a wealth of interesting physical phenomena that are increasingly used in applications. Examples are the Giant Magneto resistance effect [1, 2], the difference in resistance for parallel and antiparallel alignment of the magnetization directions of the two FM layers, which is used in magnetic read heads based on spin-valves [3]. The inverse effect, the influence of an electrical current on the magnetic configuration of the trilayer system, has drawn a lot of attention recently [4, 5, 6].

In these trilayer systems, interactions between the two FM layers through the NM spacer layer can be induced either by exchange [7], magnetostatic [8] or dynamic effects [9]. Local variations in these interlayer interactions, caused by modulated topography [10] or inhomogeneous magnetization, can be important for the static and dynamic magnetic properties of $\mathrm{FM} / \mathrm{NM} / \mathrm{FM}$ trilayers. In this paper, we will focus on the effect of domain walls in one or both FM layers, which lead to strongly localized magnetostatic interactions between the two layers. These interactions can lead to a local decrease of tle nucleation barrier for magnetization reversal 111 or to the demagnetization of one of the layers [12]. They might also play a role in the decrease of the critical current in trilayer systems for the propagation of domain walls induced by spin-polarized current [13, 14. The goal of this study was to obtain a better understanding of the interaction between magnetic layers through a nonmagnetic spacer layer in the vicinity of domain walls, using high-resolution x-ray photoemission electron microscopy combined with x-ray magnetic circular dichroism (XMCD-PEEM).

Microscopic evidence of the influence of domain wall stray fields in one layer on the static domain configuration of another layer was obtained by different groups [15, 16, 17, 18, 19]. Thomas et al. 12] showed that stray fields of domain walls sweeping back and forth during repeated switching of one of the FM layers can demagnetise the other magnetic layer, even if it is much harder magnetically. We have recently shown [11 that in nanosecond magnetization switching, domain walls in the hard magnetic Co layer of $\mathrm{Fe}_{20} \mathrm{Ni}_{80} / \mathrm{Al}_{2} \mathrm{O}_{3} / \mathrm{Co}$ trilayers can trigger nucleation in the soft FeNi layer and thereby increase the local switching speed. These observations were made taking advantage of the element selectivity, spatial and temporal resolution of time-resolved XMCD-PEEM [20, 21, 22, 23]. The high-resolution static XMCDPEEM images of $\mathrm{FeNi} / \mathrm{Al}_{2} \mathrm{O}_{3} / \mathrm{Co}$ trilayers presented in this paper nicely reveal the influence of stray fields of domain walls in the Co layer on the local magnetization of the FeNi layer. These stray fields lead to a localised antiparallel coupling between the Co and FeNi moments. For strongly charged walls, which are not parallel to the easy magnetization axis of the films, this antiparallel coupling can extend over radial distances of several micrometers from the centre of the domain wall.

\section{Experimental details}

The sample consisted of a stack of continuous layers, $\mathrm{CoO}(3 \mathrm{~nm}) / \mathrm{Co}(7 \mathrm{~nm}) / \mathrm{Al}_{2} \mathrm{O}_{3}(2.6 \mathrm{~nm})$ $/ \mathrm{Fe}_{20} \mathrm{Ni}_{80}(4 \mathrm{~nm})$, deposited on an oxidized $\mathrm{Si}(111)$ substrate. It was covered with $3 \mathrm{~nm}$ of $\mathrm{Al}$ to protect the layers from oxidation. The $\mathrm{Si}(111)$ substrate was miscut by $8^{\circ}$ along the $[11 \overline{2}]$ direction and then heat-treated to obtain a step-bunched surface, with terraces having an average width of $20 \mathrm{~nm}$ separated by $4 \mathrm{~nm}$ high steps [24]. For 
sufficiently thin films, this substrate topography is transmitted to the deposited layers [10, 25]. The presence of elongated terraces leads to a shape-induced uniaxial magnetic anisotropy with the easy magnetization axis along the long axis of the terraces. The correlated roughness at the two FM/NM interfaces leads to a magnetostatic orangepeel coupling [8] between the two magnetic layers [10, 26].

In order to obtain XMCD-PEEM images with high spatial resolution $(<40 \mathrm{~nm})$, we made use of the SPELEEM instrument at the Nanospectroscopy beamline at the synchrotron ELETTRA (Trieste, Italy) [27, 28]. In this microscope, the x-rays hit the sample surface at a grazing incidence angle of $16^{\circ}$. It is therefore particularly suited for the study of samples with in-plane magnetization. Element-selective magnetic contrast was obtained using XMCD, with the x-ray energy tuned to the maximum of the $\mathrm{Fe}_{3}$ absorption edge $(720 \mathrm{eV})$ for the FeNi layer and of the $\mathrm{Co} \mathrm{L}_{3}$-edge $(793 \mathrm{eV})$ for the Co layer. The images, obtained at room temperature, represent the asymmetry (difference divided by sum) of two measurements taken with opposite photon helicity. This allows optimizing the magnetic contrast while minimizing topographic effects.

\section{Results and discussion}

The quasi-static magnetization hysteresis loops of the sample, obtained using the longitudinal magneto-optical Kerr effect, are shown in Fig. 1. The quasi-static magnetization reversal of this sample takes place by nucleation of a small number of domains and a subsequent propagation of the domain walls [29]. The minor loops of the FeNi layer reveal a coupling strength of about $0.6 \mathrm{mT}$.

Before taking images, an AC magnetic field with decreasing amplitude was applied to the sample along the easy magnetization axis, in order to create a multidomain state in both the FeNi and Co layers. A small constant field of about $-3 \mathrm{mT}$ was then applied, higher than the FeNi coercivity but lower than the Co coercivity, in order to saturate the FeNi layer without changing the Co domain structure (Fig. 11). The sample was then introduced into the microscope, and several regions of interest were imaged. Figure 2 shows some typical magnetic domain images of the Co (a) and

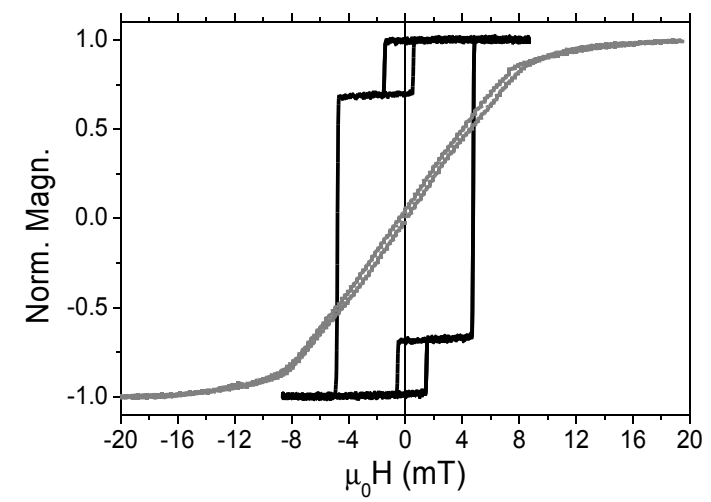

Figure 1. Quasi-static hysteresis loops of the magnetic tunnel junction-like trilayer obtained by longitudinal Kerr effect. Loops obtained with the field applied along the easy (black line) and hard (grey line) magnetization axes are shown, as well as minor loops for the FeNi layer taken along the easy axis. 


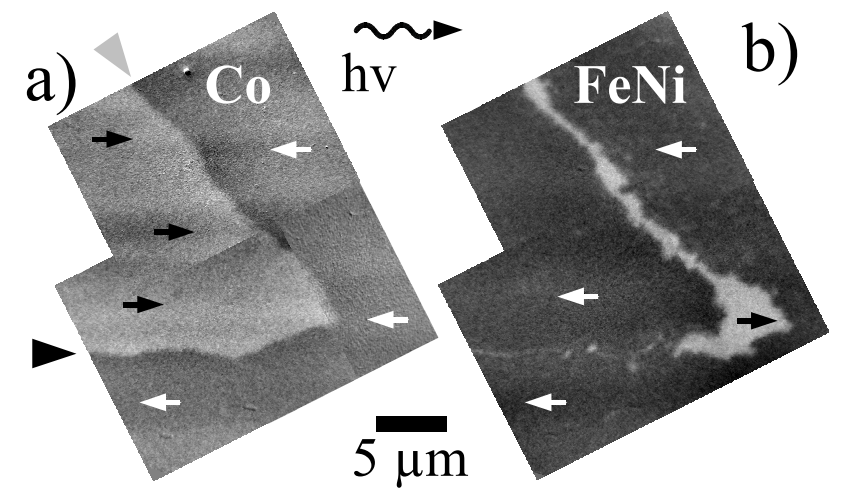

Figure 2. Magnetic domain images of the $\mathrm{Co}$ (a) and $\mathrm{FeNi}$ (b) layers, after demagnetising the sample and consecutively re-saturating the FeNi layer. The directions of local magnetization and of incoming photons are indicated with arrows. The black and grey arrowheads next to the Co image indicate, respectively, the Co domain wall oriented parallel to the easy magnetization axis and the one tilted of about $45^{\circ}$ with respect to this axis

FeNi (b) layers. The images were obtained with the projection of the x-ray incidence direction on the sample surface parallel to the easy magnetization axis. The contrast is due to the difference in absorption for domains with their magnetization direction parallel and antiparallel to the incoming x-ray direction.

The image of the Co layer shows a domain structure typical for a magnetic layer with in-plane magnetic anisotropy, with one large white domain (top left part of the image) and one large black domain. Contrast variations inside the domains are due to inhomogeneous intensity of the x-ray beam. Two main domain wall orientations are present, one parallel to the easy magnetization axis (indicated with a black arrowhead) and the other tilted with respect to this axis of about $45^{\circ}$ (grey arrowhead).

In the FeNi image, clear differences are observed between the regions above the two sections of the Co domain wall with different orientations. Above the parallel DW, a faint grey line is visible, indicating that the magnetization is tilted away from the easy axis to form a 'quasi-wall' [11, 30. For the layer thickness used here, domain walls in both Co and FeNi layers are expected to be of Néel-type. The presence of the Néel wall in the Co-layer implies a local divergence of the in-plane magnetization, leading to a magnetic charge $-\nabla \cdot \mathbf{M}$. The associated stray field points in the direction opposite to the magnetization at the centre of the Co domain wall, as indicated in Fig. 3(left), and induces the 'quasi-wall' in the FeNi layer.

In the vicinity of the tilted Co DW, much larger white domains are visible in the FeNi layer. The magnetization directions in the Co layer around this DW are partly pointing towards each other, leading to a so-called 'head-on' DW. The stray field induced by this strongly charged DW, as indicated in Fig. 3(right), is much larger than for the parallel DW. In order to partly compensate the magnetic charges on the Co DW, an oppositely charged domain wall is formed in the FeNi layer (a tail-totail domain wall). The Co DW stray field therefore locally induces an antiparallel coupling between the two magnetic layers through the alumina spacer layer, as is clearly seen in Fig. 2: the white domains in the FeNi layer are situated on the right side of the Co DW, above the black domain in the Co layer. This DW induced 
antiparallel coupling apparently is much stronger than the orange-peel coupling that favors parallel alignment.

In order to allow a better observation of the domain walls, we rotated the sample by $90^{\circ}$. The corresponding images, thus taken with the x-rays coming in along the hard magnetization axis, are shown in Fig. 1 . The azimuthal rotation was performed outside the microscope, since an in-situ rotation was not possible. This makes it almost impossible to find back exactly the same region of the sample in this kind of continuous films. The region in Fig. 1 shows therefore a domain structure that is similar to the one in Fig. 2, but not exactly the same. In this case, the observed black and white regions correspond to the domain walls or quasi-walls, while the domains show intermediate grey contrast. In the left (Co) image, a zigzag domain wall is observed as in Fig. 2, and the magnetization direction at the centre of the DW is indicated by the arrows. The expected magnetization direction inside the domains is also given. Two Bloch lines (vortices), characterised by a reversal of the direction of magnetization at the centre of the DW, can be clearly observed. They are indicated by black arrowheads in Fig. Aa).

Figure 4 b) shows that at positions where a domain wall is present in the Co layer, domain walls or quasi-walls are present also in the FeNi layer. The contrast at these positions and thus the magnetization direction at the centre of the wall is opposite in the Co and FeNi layers. This is due to the direction of the domain wall stray field, and allows a partial compensation of the domain wall charges in both layers. Micromagnetic simulations confirm this picture 11. Additional domain walls in the

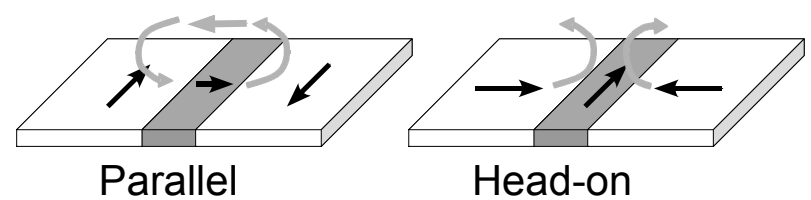

Figure 3. Schematic representation of domain wall configurations for 'parallel' (left) and 'head-on' (right) Néel-type domain walls and the associated domain wall stray fields. The black arrows indicate the local magnetization direction in the film, the grey arrows the associated stray field directions.

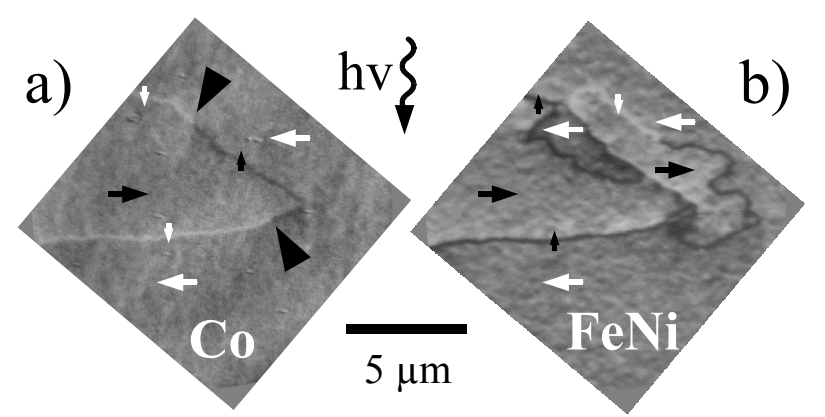

Figure 4. Magnetic domain images of the Co (a) and FeNi (b) layers, taken with the incoming x-rays perpendicular to the easy magnetization axis. The black and white regions correspond to domain walls, the grey regions to domains. The black arrowheads in the Co image indicate the positions of two Bloch lines. 

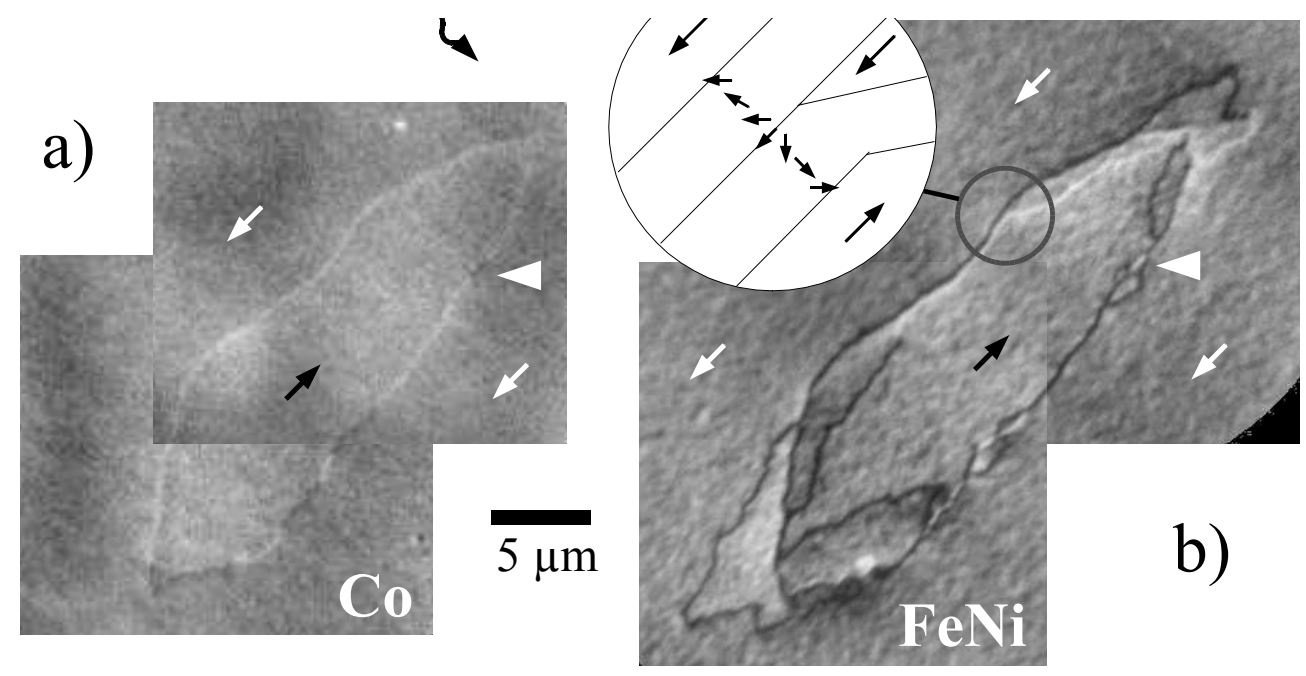

Figure 5. Magnetic domain images of the Co (a) and FeNi (b) layers. The images were taken with the incoming $\mathrm{x}$-ray perpendicular to the easy magnetization axis, leading to a contrast induced by the domain walls only. The black circle indicates a position where a $180^{\circ}$ domain wall and a quasi-wall join. A schematic representation of the magnetization directions in this region is shown in the large circle.

FeNi layer, which are not associated to the Co DW, are due to the extended domains generated in the FeNi layer by the stray field of the tilted Co DW (see Fig. 2b).

Outside the region where domain walls are present, contrast modulations are visible. These modulations are not due to noise, since they are reproducible between different series of images taken at the same position. This indicates that the local magnetization direction can deviate from the global easy magnetization axis. This is a remainder of the so-called 'ripple' structure often observed for uniaxial polycrystalline thin films when a magnetic field is applied perpendicular to the easy magnetization axis. The phenomenon can be associated to the crystalline anisotropy of individual grains [31]. In our case, it is probably associated to a modulation of the long axis of the substrate terraces with respect to the easy [110] axis. The long axis of each terrace determines the local shape anisotropy, leading to a distribution of magnetization directions around the average, macroscopic easy magnetization axis.

In Fig. 5, we show an ellipse-shaped domain structure in the Co layer, obtained after complete demagnetization of the sample. In this case, the magnetization directions in both layers are expected to be globally parallel far from the domain wall regions, due to the orange-peel coupling. The expected magnetization directions are indicated by arrows in Fig. 河. In the Co domain wall, several Bloch lines are clearly visible. At these Bloch lines, the magnetization direction at the centre of the DW reverses direction, and therefore also the emitted stray field. In most cases, the corresponding magnetization direction at the centre of the FeNi DW also changes sign. This can be seen for instance at the position indicated by the white arrowhead in Fig. 国, where the small black segment in the Co DW is accompanied by a small white segment in the FeNi DW. At the centre of the Bloch lines, the magnetization direction is perpendicular to the plane of the film [32, leading to the presence of additional 
stray field components. This leads to a more complex magnetization configuration in the FeNi film close to the Bloch lines.

The strongly charged domain walls at the extremities of the ellipse-shaped Co domain induce small domains in the FeNi layer at both sides of the Co DW. As mentioned above, the magnetic charges in the Co layer can be partly compensated by creating charges of opposite sign in the FeNi layer. This leads to a strong antiparallel coupling between $\mathrm{Co}$ and $\mathrm{FeNi}$ layers close to the charged Co domain walls. This influence of the domain wall charges is felt up to lateral distances of several micrometers from the domain wall centre.

At several positions, a grey/black/white/grey contrast can be seen around domain walls in the FeNi layer. This is what is expected for a $360^{\circ}$ domain wall, where the magnetization vector makes a complete $360^{\circ}$ turn going from one side of the domain wall to the other. However, also other configurations can give rise to this contrast. As an example consider the region in the circle of the FeNi image of Fig. 同. On the lower left, a black/white contrast is present. Going to the upper right, the black and white parts split. The white part is not associated to a DW in the Co layer, indicating that it has to be a normal $180^{\circ}$ Néel wall. The black part separates two domains with the same magnetization direction and is therefore a 'quasi-wall', induced by the stray field of the Co domain wall. This means that, crossing the black/white region from lower right to upper left, the magnetization vector turns over an angle of about $270^{\circ}$, before turning back $-90^{\circ}$ to end up at an angle of $180^{\circ}$ with respect to the initial magnetization direction. The contrast is thus induced by the combination of a Néel wall and a 'quasi-wall' with the same chirality. A zoom of the magnetic configuration is schematically shown in the circle.

These images can be used to obtain an estimate of the domain wall width in both layers. The definition we use for determining the domain wall width is based on the total wall flux and is given by $W_{F}=\int_{-\infty}^{\infty} \cos \varphi(x) d x$ [30], where $\varphi(x)$ is the angle between the local magnetization direction and the hard magnetization axis. This definition is the most convenient in our case, since the XMCD-intensity in the images of Fig. 团 is proportional to the projection of the local magnetization direction on the xray incidence direction and thus to $\cos \varphi$. The wall width can be obtained by taking an image profile along a direction perpendicular to the DW. Using the highest resolution images (field of view $10 \mu \mathrm{m}$ ), we find widths of $130 \pm 20 \mathrm{~nm}$ for the Co domain walls and $170 \pm 15 \mathrm{~nm}$ for the FeNi domain walls. However, the domain wall widths seem to be quite irregular, probably due to the specific, irregular topography of terraces and steps that can locally lead to confinement or, on the contrary, largening of the domain walls [10]. The DW width of Co is smaller than for FeNi. According to Ref. [30], Chap. 5.5.7, the Néel wall width in each layer of a magnetic/non-magnetic/magnetic trilayer system is determined by the magnetic anisotropy $K_{u}$ and the stray field energy (with parameter $K_{D}=J_{S}^{2} / \mu_{0}$, where $J_{S}$ is the spontaneous magnetization). While the magnetic anisotropy tends to decrease the domain wall width, the stray field energy tends to increase it. In our case, the magnetic anisotropy is larger for the Co layer $\left(K_{U}^{C o} \approx 11200 J / m^{3}\right.$ against $K_{U}^{F e N i} \approx 1720 J / m^{3}$ (Ref. [11]) but also the exchange and the stray field energy $\left(J_{S}^{C o}=1.76 T, J_{S}^{F e N i}=1 T\right)$. The wall width of the Co domain walls is not much smaller than the one of the FeNi layer, but the stray field energy is about three times higher. Moreover, it is easier to deviate the FeNi magnetization from its easy axis due to the smaller anistropy. The influence of the Co DW stray field on the FeNi layer is therefore much larger than the reciprocal effect, which is however still visible in some images (see Fig. 四a, for example). 


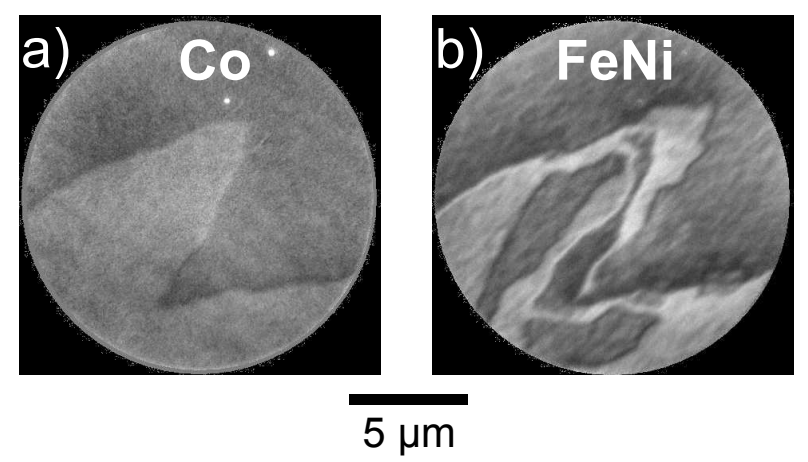

Figure 6. Magnetic domain images of the Co (a) and FeNi (b) layers after application of several bipolar pulses with an amplitude of $5 \mathrm{mT}$. The images were taken with the x-rays coming in at an azimuthal angle of $10^{\circ}$ with respect to the hard magnetization axis.

Time-resolved XMCD-PEEM measurements on this same sample already revealed the strong influence of the Co domain walls on the magnetization reversal of the FeNi layer (Fig. 7 of Ref. 29]). In that case, a non-saturated region was observed in the FeNi layer just above the Co domain wall even for fields of $6 \mathrm{mT}$. This region seemed to be rather large (about $1 \mu \mathrm{m}$ ), larger than we observe in these static images, even taking into account the difference in spatial resolution (about $50 \mathrm{~nm}$ here against 300 nm in Ref. 29). In order to investigate the influence of repeated magnetic pulses on the domain walls, we recorded images of the Co and FeNi layers after application of some thousands of bipolar magnetic pulses of about $5 \mathrm{mT}$, using the same coil and pulsed current supply as in Refs. 29, 33]. The corresponding images are shown in Fig. 6.

The images were recorded with the x-rays coming in at an azimuthal angle of $80^{\circ}$ with respect to the easy magnetization axis. In this way it is possible to reveal the magnetization direction in both the domains and the domain walls. The FeNi image clearly shows signs of domain accumulation around the Co domain wall over distances well above $1 \mu \mathrm{m}$. Compression of these domains during the application of the field pulses might lead to $360^{\circ}$ domain walls and be at the origin of the larger grey region observed in the images of Ref. [29].

\section{Conclusion}

We have performed layer-selective imaging of magnetic domains in $\mathrm{FeNi} / \mathrm{Al}_{2} \mathrm{O}_{3} / \mathrm{Co}$ trilayers with high spatial resolution. These images reveal the strong interaction between domain walls in the two magnetic layers. Co domain walls parallel to the easy magnetization axis induce 'quasi-walls' in the FeNi layer, with the magnetization at the centres of the two walls in opposite directions. For domain walls that are tilted with respect to the easy magnetization axis magnetostatic interactions favor an anti-parallel alignment of the magnetization directions in both magnetic layers over a distance up to several microns from the domain wall. Upon repetitive application of magnetic field pulses, these interactions can lead to an accumulation of domains and domain walls in the FeNi layer around the Co DW positions. 


\section{Acknowledgments}

We acknowledge partial funding by the European Union under contract No. HPRNCT-2000-00134 and by a Programme d'Actions Intégrées 'Picasso' (J.V. and J.C.), through Grant No. HF2003-0173. We thank A. Vaurès for her invaluable help in sample preparation.

\section{References}

[1] Baibich M N, Broto J M, Fert A, Nguyen Van Dau F, Petroff F, Etienne P, Creuzet G, Friederich A and Chazelas J 1988 Giant magnetoresistance of $(001) \mathrm{Fe} /(001) \mathrm{Cr}$ magnetic superlattices Phys. Rev. Lett. 612472

[2] Binasch G, Grünberg P, Saurenbach F and Zinn W 1989 Enhanced magnetoresistance in layered magnetic structures with antiferromagnetic interlayer exchange Phys. Rev. B 394828

[3] Dieny B, Speriosu V S, Parkin S S P, Gurney B A, Wilhoit D R and Mauri D 1991 Giant magnetoresistive in soft ferromagnetic multilayers Phys. Rev. B 431297

[4] Slonczewski J 1996 Current-driven excitation of magnetic multilayers J. Magn. Magn. Mater. $159 \mathrm{~L} 1$

[5] Myers E B, Ralph D C, Katine J A, Louie R N and Buhrman R A 1999 Current-induced switching of domains in magnetic multilayer devices Science $\mathbf{2 8 5} 867$

[6] Katine J A, Albert F J, Buhrman R A, Myers E B and Ralph D C 2000 Current-driven magnetization reversal and spin-wave excitations in co/cu/co pillars Phys. Rev. Lett. 84 3149

[7] Bruno P and Chappert C 1992 Ruderman-kittel theory of oscillatory interlayer exchange coupling Phys. Rev. B 46261

[8] Néel L 1962 Sur un nouveau mode de couplage entre les aimantations de deux films $C$. $R$. Hebd. Seances Acad. Sci. 2551676

[9] Heinrich B, Tserkovnyak Y, Woltersdorf G, Brataas A, Radovan U and Bauer G E 2003 Dynamic exchange coupling in magnetic bilayers Phys. Rev. Lett. 90187601

[10] Pennec Y, Camarero J, Toussaint J C, Pizzini S, Bonfim M, Petroff F, Kuch W, Offi F, Fukumoto K, Nguyen Van Dau F and Vogel J 2004 Switching mode dependent magnetic interlayer coupling strength in spin-valves and magnetic tunnel junctions Phys. Rev. B 69180402

[11] Vogel J, Kuch W, Hertel R, Camarero J, Fukumoto K, Romanens F, Pizzini S, Bonfim M, Petroff F, Fontaine A and Kirschner J 2005 Influence of domain wall interactions on nanosecond switching in magnetic tunnel junctions Phys. Rev. B 72220402

[12] Thomas L, Samant M G and Parkin S S P 2000 Domain-wall induced coupling between ferromagnetic layers Phys. Rev. Lett. 84001816

[13] Grollier J, Boulenc P, Cros V, Hamzić A, Vaurès A, Fert A and Faini G 2003 Switching a spin valve back and forth by current-induced domain wall motion Appl. Phys. Lett. 83509

[14] Laribi S, Cros V, Muñoz M, Grollier J, Hamzić A, Deranlot C, Fert A, Martínez E, López-Díaz L, Vila L, Faini G, Zoll S and Fournel R 2007 Reversible and irreversible current induced domain wall motion in cofeb based spin valves stripes Appl. Phys. Lett. 90232505

[15] Fuller H W and Sullivan D L 1962 Magnetostatic interactions between thin magnetic films $J$. Appl. Phys. 331063

[16] Kuch W, Chelaru L I, Fukumoto K, Porrati F, Offi F, Kotsugi M and Kirschner J 2003 Layerresolved imaging of magnetic interlayer coupling by domain-wall stray fields Phys. Rev. B 67214403

[17] Schäfer R, Urban R, Ullmann D, Meyerheim H L, Heinrich B, Schultz L and Kirschner J 2002 Domain wall induced switching of whisker-based tunnel junctions Phys. Rev. B 65144405

[18] Christoph V and Schäfer R 2004 Numerical simulation of domain walls in fe whiskers and their interaction with deposited thin films Phys. Rev. B 70214419

[19] Wiebel S, Jamet J P, Vernier N, Mougin A, Ferré J, Baltz V, Rodmacq B and Dieny B 2005 Domain decoration in dipolar coupled ferromagnetic stacks with perpendicular anisotropy Appl. Phys. Lett. 86142502

[20] Kuch W 2004 X-ray magnetic circular dichroism for the quantitative element-resolved magnetic microscopy Phys. Scr. T109 89

[21] Vogel J, Kuch W, Bonfim M, Camarero J, Pennec Y, Offi F, Fukumoto K, Kirschner J, Fontaine A and Pizzini S 2003 Time-resolved magnetic domain imaging by x-ray photoemission electron microscopy Appl. Phys. Lett. 822299 
[22] Bonfim M, Ghiringhelli G, Montaigne F, Pizzini S, Brookes N B, Petroff F, Vogel J, Camarero $\mathrm{J}$ and Fontaine A 2001 Element-selective nanosecond magnetization dynamics in magnetic heterostructures Phys. Rev. Lett. 863646

[23] Schneider C M, Krasyuk A, Nepijko S A, Oelsner A and Schönhense G 2006 Accessing fast magnetization dynamics by xpeem: Status and perspectives J. Magn. Magn. Mater. 3046

[24] Sussiau M, Nguyen Van Dau F, Galtier P, Encinas A and Schuhl A 1997 Bicrystalline magnetic lateral superlattices J. Magn. Magn. Mater. 1651

[25] Montaigne F 1999 Effet tunnel dépendant du spin : Des simples aux doubles jonctions. PhD thesis Université Paris VII - Denis Diderot

[26] Encinas A, Nguyen Van Dau F, Schuhl A, Montaigne F, Sussiau M and Galtier P 1999 Properties of spin-valve structures deposited on step-bunched vicinal surfaces J. Magn. Magn. Mater. 98-99 15

[27] Locatelli A, Bianco A, Cocco D, Cherifi S, Heun S, Marsi M, Pasqualetto M and Bauer E 2003 High lateral resolution spectroscopic imaging of surfaces: The undulator beamline "nanospectroscopy" at ELETTRA J. Phys. IV $\mathbf{1 0 4} 99$

[28] Locatelli A, Aballe L, Mentes T, Kiskinova M and Bauer E 2006 Photoemission electron microscopy with chemical sensitivity: Speleem methods and applications Surf. Interf. Anal. 381554

[29] Romanens F, Vogel J, Kuch W, Fukumoto F, Camarero J, Pizzini S, Bonfim M and Petroff F 2006 Influence of topography and Co domain walls on the magnetization reversal of the FeNi layer in FeNi/Al2O3/Co magnetic tunnel junctions Phys. Rev. B $\mathbf{7 4} 184419$

[30] Hubert A and Schäfer R 1998 Magnetic Domains : The Analysis of Magnetic Microstructures (Berlin: Springer)

[31] Harte K J 1968 Theory of magnetization ripple in ferromagnetic films J. Appl. Phys. 391503

[32] Wachowiak A, Wiebe J, Bode M, Pietzsch O, Morgenstern M and Wiesendanger R 2002 Direct observation of internal spin structure of magnetic vortex cores Science 29857

[33] Vogel J, Kuch W, Camarero J, Fukumoto K, Pennec Y, Bonfim M, Pizzini S, Petroff F, Fontaine A and Kirschner J 2004 Time and layer resolved magnetic domain imaging of $\mathrm{FeNi} / \mathrm{Cu} / \mathrm{Co}$ trilayers using x-ray photoelectron emission microscopy J. Appl. Phys. 956533 